\title{
Nuevo dato sobre la longevidad del murciélago grande de herradura Rhinolophus ferrumequinum (Schreber, 1774) en las islas Baleares
}

\author{
David García ${ }^{1 *}$, Llorenç CAPEllì̀ ${ }^{2} \&$ JuAn Quetglas ${ }^{3}$
}

\author{
${ }^{1} \mathrm{C} /$ Son Borràs, 14. 07340 Alaró. Mallorca. Islas Baleares (Spain) \\ ${ }^{2}$ C/ Plaça Nova, 3007730 - Alaior. Menorca Islas Baleares (Spain) \\ ${ }^{3}$ Urb. El Señorío 25, 41807- Espartinas, Sevilla (Spain) \\ *Correo electronico del autor: baldritja@yahoo.es \\ DOI: http://dx.doi.org/10.14709/BarbJ.6.1.2013.08
}

English title: New data of longevity in the Greater Horseshoe bat Rhinolophus ferrumequinum (Schreber, 1774) in the Balearic Islands

\begin{abstract}
In this note we report the capture of a greater horse-shoe bat (Rinolophus ferrumequinum) ringed 16 years ago in Menorca Island, resulting in the oldest bat ever known from the Balearic Islands. This recapture is an important contribution to the understanding of bat longevity in insular environments.

Keywords: Greater Horseshoe bat, Rhinolophus ferrumequinum, longevity, Balearic Islands.

Resumen: Se captura un ejemplar de Rhinolophus ferrumequinum que fue anillado hace 16 años, tratándose del murciélago más longevo registrado en el Archipiélago Balear. Esta aportación contribuye al conocimiento de la longevidad de quirópteros en ámbitos insulares.
\end{abstract}

Palabras claves: Murciélago grande de herradura, Rhinolophus ferrumequinum, longevidad, Islas Baleares.

Los murciélagos constituyen el grupo de mamíferos que alcanza una mayor longevidad en proporción a su tamaño (Bouliere 1958; Austad \& Fischer 1991). Entre las diferentes hipótesis que se barajan para explicar estos altos niveles de longevidad están: un bajo índice de bajas causadas por depredadores, gracias a la capacidad de poder esquivarlos mediante el dominio del vuelo; un reducido costo reproductor y a la restricción calórica que supone la hibernación (Wilkinson \& South 2002; Brunet-Rossinni \& Austad 2004). El conocimiento de la esperanza de vida de los quirópteros va mejorando a lo largo de las últimos años gracias a la aportaciones proporcionadas por las recapturas de animales marcados con anillas, por lo que este conocimiento sobre la esperanza de vida de muchas especies está en un continuo cambio (Brunet-Rossinni \& Austad 2004).

En la bibliografía específica se han documentado casos de longevidad en diversas especies de quirópteros (ver ejemplo: Lehmann et al. 1992; Davis \& Hitchcock 1995; Ibánez \& Pérez-Jordá 1998), siendo el máximo registro de longevidad actual de un murciélago silvestre de 38 años, Myotis brandtii (Vespertilionidae) en Siberia (Khritankov \& Ovodov 2001), mientras que en Europa el murciélago más longevo hallado ha sido un Myotis blythii (Vespertilionidae) con 33 años (Arlettaz et al. 2002). En Baleares la edad más elevada constatada en quirópteros hasta la actualidad es de un ejemplar de Myotis myotis 


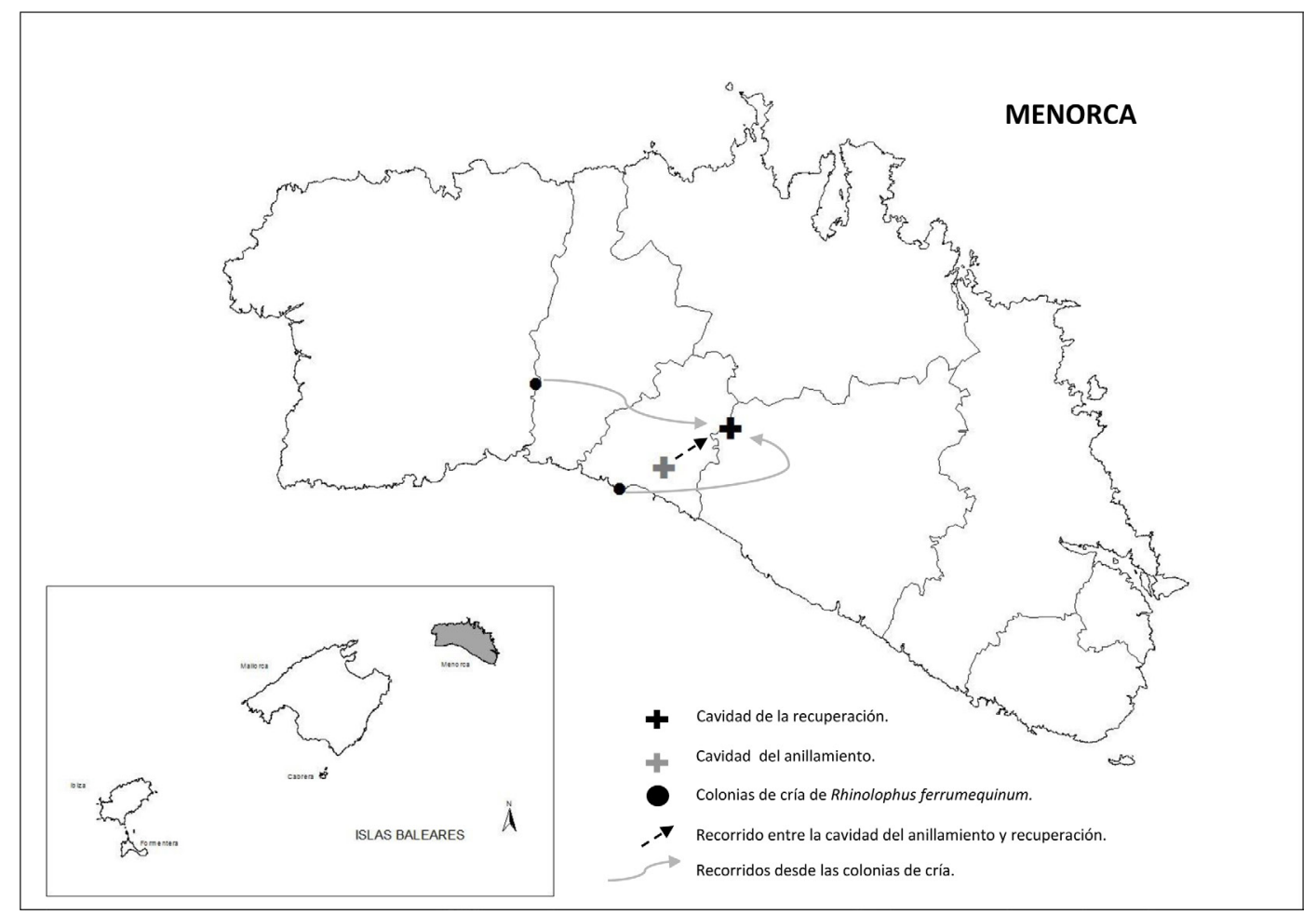

Fig. 1 Localización de las cavidades donde fue anillado y recapturado el murciélago grande de herradura (Rhinolophus ferrumequinum) y los posibles recorridos desde las colonias de cría.

(Vespertilionidae) con 9 años (Serra-Cobo et al. 2007). En el caso de Rhinolophus ferrumequinum se ha registrado un máximo de 30,5 años (Caubère et al. 1984), siendo habitual la presencia de ejemplares con una edad aproximada de 15 años en las colonias de este rinolófido (Dietz et al. 2009). Cabe mencionar, que en muchas de las referencias bibliográficas de longevidad de quirópteros no se tenía un conocimiento previo de la edad de los ejemplares anillados, por lo que es de esperar que en muchos casos la edad del animal supere al periodo transcurrido de la recuperación.

Rhinolophus ferrumequinum está presente en Mallorca, y Menorca (Alcover \& Muntaner 1986), y en 2004 fue constatada su presencia en Formentera, donde se consideraba extinta (Alcover \& Muntaner 1986), estimándose una población entre 20 - 40 ejemplares (Trujillo et al. 2005). La especie está extinta de Ibiza, donde solo se conoce su presencia en el registro fósil (Alcover 2003). $\mathrm{Su}$ población se ha estimado entorno a los 6001.000 ejemplares en el conjunto del archipiélago (García et al. 2012), agrupándose gran parte de sus efectivos en unas pocas colonias de Menorca, (lo que supone un factor de riesgo adicional para su población), siendo cada una de estas concentraciones de individuos de gran importancia para la dinámica poblacional y supervivencia de la especie.
Entre 1995 y 2000 se marcaron con anillas con remite de ICONA unos 446 ejemplares de murciélagos correspondiente a ocho especies: Rhinolophus ferrumequinum (36); Myotis capaccinii (34); Myotis escalerai (29); Myotis emarginatus (10); Pipistrellus pipistrellus (15); Pipistrellus kuhlii (26); Hypsugo savii (15); Miniopterus schreibersii (281). Durante una jornada de trampeo el 26 de septiembre de 2012 se recapturó un macho de Rhinolophus ferrumequinum en una trampa arpa en una cavidad en Menorca cuando pretendía entrar, sobre las 20:20h. Gracias a la base de datos de anillamientos de la SECEMU, se pudo comprobar que dicho murciélago había sido anillado el 5 de abril de 1996 (por el tercer autor de esta nota), hacía 16 años, cinco meses y veintiún días. El rinolofo grande fue anillado en una cavidad que es empleada por la especie como refugio nocturno, a una distancia de $3,7 \mathrm{~km}$ de la cueva donde ha sido recuperado. Cabe señalar que la distancia entre las dos cavidades de cría conocidas en la actualidad en Menorca y la localidad donde fue recuperado este rinolófido es de $9,5 \mathrm{~km}$ y 6,07 respectivamente, siendo muy probable que el animal provenga de estos refugios (ver figura 1).

Aunque la longevidad proporciona escasa información sobre la supervivencia (Krementz et al. 1989), es necesario poder contar actualmente 
con esta información para comprender la dinámica poblacional de algunas especies de quirópteros cavernícolas en el archipiélago. Por otro lado, las incidencias de anillas pueden ocasionar heridas de diferente índole (ver ejemplos: Dietz et al. 2006; Zambelli et al. 2009), llegando a afectar a la supervivencia de los animales marcados (Zambelli et al. 2009) por lo que su uso se ha restringido en la actualidad notablemente tanto en relación al número de especies como al de individuos que se anillaban en tiempos anteriores. Aunque, es recomendable conocer el grado de incidencias del anillamiento sobre esta y otras especies.

La recaptura que se presenta contribuye enormemente a mejorar los conocimientos sobre la longevidad de la fauna quiropterológica en un ámbito insular como es el caso de Baleares, donde se dispone de muy escasos registros de ejemplares longevos. Sin embargo, es necesario mejorar la información actual sobre las tasas de supervivencia de esta especie en las islas, así como mejorar las estimas poblacionales de esta especie, lo que sin duda facilitará la toma de decisiones adecuada en relación a garantizar su conservación en el Archipiélago Balear .

\section{Agradecimientos}

Queremos expresar nuestro agradecimiento a Óscar de Paz y Domingo Trujillo por aportarnos algunas reseñas bibliografía que han sido de mucha utilidad. También agradecer a Patricia Arbona y Javier Juste la revisión de este manuscrito.

\section{REFERENCIAS}

Alcover, J. A. \& Muntaner, J. 1986. Els quiròpters de les Balears i Pitiüses: una revisió. Endins, 12: 51-63.

Alcover, J.A. 2003.Les rates pinyades(Mammalia: Chiroptera) Fòssils del jaciment paleontògic del Pouàs (St. Antoni de Portmany, Eivissa). Endins, 25: 141-154.

Arlettaz, R., Christe, P. \& Desfayes, M. 2002. 33 years, a new longevity record for a European bat. Mammalia, 66: 441-442.
Austad, S.N. \& Fischer, K.E. 1991. Mammalian ageing, metabolism and ecology: evidence from the bats and marsupials. J. Gerontol, 46 (2): 47-53. DOI: https://dx.doi.org/10.1093/ geronj/46.2.B47

Bourliere, M.D. 1958. The comparative biology of aging. J. Gerontol, 13: 16-24.

Brunet-Rossinni,A.K.\&Austad,S.N.2004.Ageing studies on bats: a review. Biogerontology, 5: 211-222. DOI: https://dx.doi.org/10.1023/ B:BGEN.0000038022.65024.d8

Caubère, B., Gaucher, P. \& Julien, J. F. 1984. Un record mundial de longevite in natura pour un Chiroptere insectivore?. Rev. Ecol. (Terre Vie) Vol., 39: 351-353.

Davis, W. H. \& Hitchcock, H. B. 1995. A new longevity record for the bat Myotis lucifugus. Bat research news, 36: 6.

Dietz C., Helversen, O. \& Nill, D. 2009. Bats of Britain, Europe \& Northwest Africa. A \& C Black Publishers Ltd.

Dietz, C., Dietz, I., Ivanova, T. \& Siemers, B.M. 2006. Effects of forearm band son horseshoe bats (Chiroptera: Rhinolophidae). Acta Chiropterologica,8(2):523-535.DOI:https:// dx.doi.org/10.3161/150811006779398591

García, D., Trujillo, D.,Arbona, P. CAPellà, Ll., Carrasco, G \& Oliver, J. 2012. Síntesis de la situación actual de las poblaciones de quirópteros cavernícolas en Baleares. Póster. IV Jornadas de SECEMU. Granollers.

IBÁÑEZ C. \& PÉREZ-JORDÁ, L. 1998. Longevity in the European free-tailed bat (Tadarida teniotis). J. Zool. Lond., 245: 213-214. DOI: https:// dx.doi.org/10.1017/S0952836998236090

Khritankov, A.M. \& Ovodov, N.D. 2001. Longevity of Brandt's bats (Myotis brandii Eversmann) in Central Siberia. Plecotus, 4: 20-24.

KrementZ, D. G., SAUER, J.R. \& Nichols, J.D. 1989. Model-based estimates of annual survival rate are preferable to observed maximum lifespan statistics for use in comparative lifehistory studies. Oikos, 56, 203-208. 
Lehmann, J., Jenni, L. \& Maumary, L. 1992. A new longevity record for the long-eared bat (Plecotus auritus, Chiroptera). Mammalia, 56: 316-318. DOI: https://dx.doi. org/10.1515/mamm-1992-0224

Serra- Cobo, Amengual, B., López-Roig, M., MÁrquez, J., Torres, M., Ripoll, A., Sánchez, A. \& Oliver, J. A. 2007. Catorze anys d'estudis quiropterològics a les Illes Balears (1993-2006). Boll. Soc. His. Nat. Balears, 49: 89-107.

Trujillo, D., García, D. \& Quetgas, J. 2005. Estatus, distribución y medidas de conservación de los quirópteros en la isla de Formentera. Direcció General de Caça, Protecció d'Espècies i Educació Ambiental. Conselleria de Medi Ambient. Govern de les Illes Balears.
Wilkinson, G.S. \& SouTH, J.M. 2002. Life history, ecology and longevity in bats. Aging Cell, 1 : 124-131.

Zambelli, N., Moretti, M. \& Bontadina, F. 2009. Negative consequences of forearm bands that are too small for bats. Acta Chiropterologica, 11 (1): 216-219. DOI: http://dx.doi. org/10.3161/150811009X465857 